\title{
Alarmierender Vorbote \\ Diabetes wegen Babybauch? Da kommt gern was nach!
}

\begin{abstract}
Gestationsdiabetes (GDM) ist in Deutschland häufig - und wird immer häufiger. So wurde im Jahr 2002 bei 8806 Frauen Schwangerschaftsdiabetes diagnostiziert, im Jahr 2008 waren es bereits 22.169 und damit 3,4\% aller Schwangeren. GDM ist nicht nur ein Risiko für Mutter und Kind, nach der Geburt haben betroffene Mütter auch ein hohes Risiko für einen manifesten Diabetes. Das belegen Ergebnisse der deutschen prospektiven GDM-Studie.
\end{abstract}

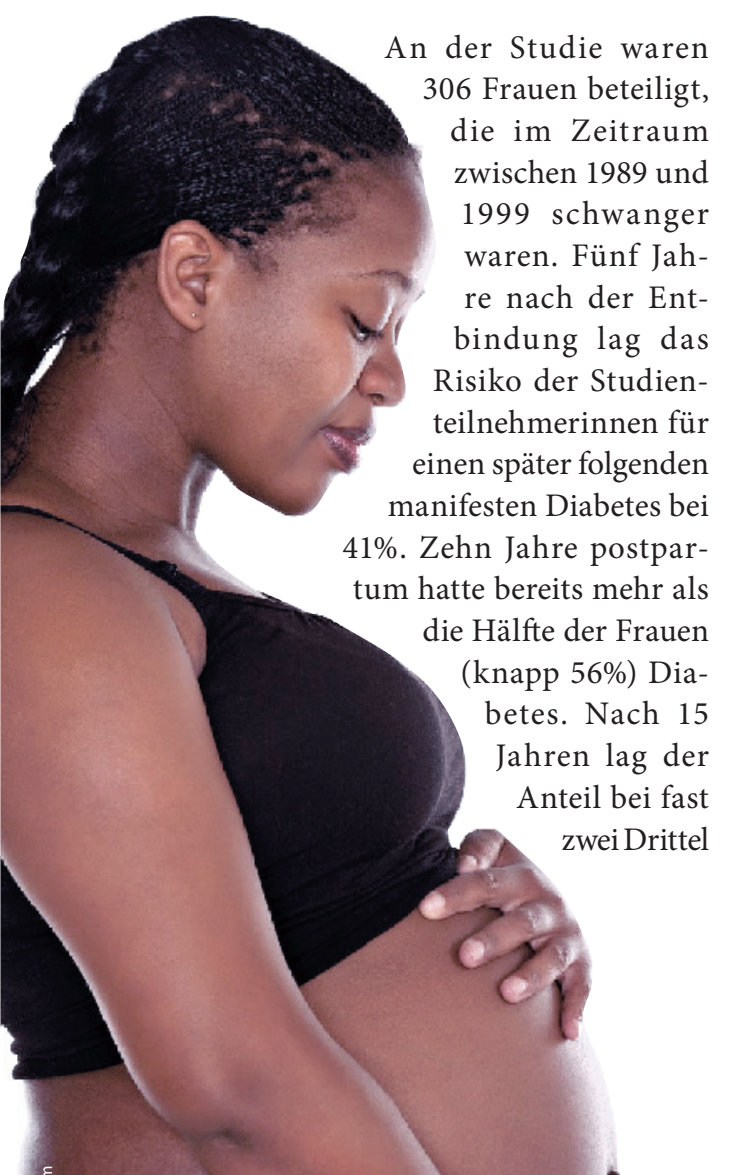

(64\%). Risikofaktoren für die DiabetesEntwicklung waren Übergewicht, eine Insulintherapie während der Schwangerschaft und der Inselantikörperstatus.

\section{Mütter regelmäßig auf Diabetes testen}

Aus diesen Ergebnissen folgt die Überlegung, dass Mütter - besonders auch nach einem Gestationsdiabetes - regelmäßig auf manifesten Diabetes untersucht werden, forderte Dr. Helmut Kleinwechter aus Kiel beim „Diabetes Update“ in Düsseldorf.

Dass Betroffene nach der Geburt mit gesunder Kost und Bewegung oder auch mit Metformin dem Diabetes erfolgreich vorbeugen können, hat eine Subgruppenanalyse des Diabetes Prevention Programms (DPP) ergeben. An dieser US-Studie hatten insgesamt 3234 Menschen ohne manifesten Diabetes aber mit gestörter Glukosetoleranz teilgenommen. Es gab dabei mehrere Interventionsgruppen, und zwar mit:

- intensiver Schulung zu Lebensstiländerung mit kalorien- und fettreduzierter Ernährung und mindestens 150 Minuten Bewegung pro Woche,

Migrantinnen haben häufig schon vor der Schwangerschaft einen Typ-2-Diabetes. Deshalb gilt hier: früh kontrollieren!
- Prävention mit Metformin oder - Behandlung mit einem Placebo.

Bei den Teilnehmern wurden im Mittel drei-Jahres-Daten erhoben. Analysiert wurden die Daten von 350 Teilnehmerinnen mit Gestationsdiabetes in der Anamnese. Der Schwangerschaftsdiabetes hatte bei den Frauen dabei im Mittel zwölf Jahre zurückgelegen.

Ergebnis: Bei den Teilnehmerinnen der Placebo-Gruppe betrug die Rate von Diabetes-Neuerkrankungen 15,4\% pro 100 Patientenjahre. In den Interventions-Gruppen waren diese Raten nur etwa halb so hoch. Ermittelt wurden 7,8\% (Metformin) und 7,4\% (Lebensstilgruppe). „In der Lebensstilgruppe mussten nur etwa fünf Frauen geschult werden, um binnen drei Jahren eine Diabetes-Erkrankung zu verhindern.“, betonte Kleinwechter.

\section{Doppelt so viele Totgeburten}

Kleinwechter wies zudem darauf hin, dass zunehmend Frauen - und zwar häufig Migrantinnen - bereits vor der Schwangerschaft an Typ-2-Diabetes leiden und damit ein hohes Risiko haben, ihr Kind zu verlieren. Nach seinen Angaben ist bei ihnen die Rate von schweren Fehlbildungen, Totgeburten und neonatalem Tod mit über $5 \%$ doppelt so hoch wie in der weiblichen Allgemeinbevölkerung.

Ärzte sollten generell Diabetikerinnen im gebärfähigen Alter über die Problematik beraten. Betroffene Schwangere sollten zum Spezialisten überwiesen werden, um den Stoffwechsel gut einzustellen und die Entwicklung des Kindes zu überwachen.

(eis) 